\title{
Investigação Citogenômica de Crianças com Doença Cardíaca Congênita: Experiência de um Centro no Brasil
}

\author{
Cytogenomics Investigation of Infants with Congenital Heart Disease: Experience of a Brazilian Center \\ Marcília Sierro Grassi, ${ }^{1}$ 닌 Marilia Montenegro, ${ }^{2}$ Evelin Aline Zanardo, ${ }^{2}$ Antonio Carlos Pastorino, ${ }^{1}$ Mayra Barros \\ Dorna, ${ }^{1}$ Chong Kim, ${ }^{2}$ Marcelo Jatene, ${ }^{1}$ Nana Miura, ${ }^{1}$ Leslie Kulikowski, ${ }^{2}$ Magda Carneiro-Sampaio ${ }^{1}$ \\ Universidade de São Paulo Hospital das Clínicas, ${ }^{1}$ São Paulo, SP - Brasil \\ Universidade de São Paulo, ${ }^{2}$ São Paulo, SP - Brasil
}

\section{Resumo}

Fundamento: Algumas síndromes têm características específicas e facilmente reconhecíveis, enquanto outras podem ser mais complexas de se identificar e podem apresentar diferentes manifestações fenotípicas, por exemplo. Um diagnóstico etiológico é importante para entender a natureza da doença, para estabelecer o prognóstico e para começar o tratamento, permitindo a inclusão de pacientes na sociedade e reduzindo o custo financeiro dessas doenças.

Objetivo: A proposta inicial deste estudo foi a triagem citogenética para detectar a síndrome de deleção $22 q 11.2$ (SD22q11.2) em recém-nascidos e crianças com doença cardíaca congênita utilizando a técnica da amplificação multiplex de sondas dependente de ligação (MLPA). Assim, por meio da pesquisa, outras mudanças genômicas foram identificadas nesses pacientes cardíacos. Nosso objetivo se estendeu a investigar essas outras mudanças citogenéticas.

Métodos: Investigamos 118 recém-nascidos com doenças cardíacas congênitas nascidos consecutivamente durante um ano, utilizando a técnica da MLPA.

Resultados: A técnica da MLPA permitiu a detecção da SD22q11.2 em 10/118 pacientes (8,5\%). Outras alterações genômicas foram identificadas em 6/118 pacientes (5\%): 1p36 del, 8p23 del (2 casos), 7q dup, 12 dup e 8q24 dup.

Conclusão: Este estudo ressalta a relevância da detecção de alterações genômicas que estão presentes em recém-nascidos e crianças com doenças cardíacas congênitas por meio de ferramentas citogenômicas.

Palavras-chave: Cardiopatias Congênitas; Anormalidades Congênitas; Diagnóstico Precoce; Síndrome de DiGeorge; Deleção Cromossômica; Recém-Nascido.

\begin{abstract}
Background: Some syndromes have specific and easily recognizable features, while others may be more complex to identify and may present different phenotypic manifestations, for example. An etiological diagnosis is important to understand the nature of the disease, to establish the prognosis and to start the treatment, allowing the inclusion of patients in society and reducing the financial cost of such diseases.

Objective: The initial proposal of this study was cytogenetic screening for the detection of the 22q11.2 deletion syndrome in consecutive newborns and infants with congenital heart disease using the multiplex ligation-dependent probe amplification (MLPA) technique. Therefore, throughout our research, other genomic alterations were identified in these cardiac patients. Thus, our objective was extended to investigate these other cytogenetic alterations.
\end{abstract}

Methods: We investigated 118 neonates with congenital heart diseases born consecutively during one year using the MLPA technique.

Results: The MLPA technique allowed the detection of 22q11.2DS in 10/118 patients (8.5\%). Other genomic alterations were also identified in 6/118 patients (5\%): $1 p 36$ del, 8p23 del (2 cases), $7 q$ dup, 12 dup and $8 q 24$ dup.

Conclusion: This study highlights the relevance of detecting genomic alterations that are present in newborns and infants with congenital cardiac diseases using cytogenomic tools.

Keywords: Heart Defects, Congenital; Congenital Abnormalities; Early Diagnosis; DiGeorge Syndrome; Chromosome Deletion; Newborn.

Full texts in English - http://www.arquivosonline.com.br

Correspondência: Marcília Sierro Grassi •

Universidade de São Paulo Hospital das Clínicas - Pediatria - Avenida Dr. Enéas de Carvalho Aguiar, 647. CEP 05403-000, São Paulo, SP - Brasil E-mail: marcilia.grassi@hc.fm.usp.br

Artigo recebido em 03/01/2020, revisado em 13/12/2020, aceito em 24/02/2021

DOI: https://doi.org/10.36660/abc.20190894 


\section{Introdução}

As anomalias congênitas são detectadas em aproximadamente $6 \%$ dos recém-nascidos - a literatura internacional descreve que 7,9 milhões de crianças nascem com doenças congênitas graves por ano. No Brasil, as malformações representam a segunda principal causa de mortalidade infantil. ${ }^{1}$

A doença cardíaca congênita (DCC), de acordo com a definição proposta por Mitchell et al., consiste de uma anomalia estrutural macroscópica do coração ou dos grandes vasos intratorácicos com repercussões funcionais significativas ou potencialmente significativas. Representa aproximadamente $40 \%$ de todas as malformações congênitas. De acordo com a Organização Mundial da Saúde (OMS), sua incidência varia de $0,8 \%$ em países de alta renda a 1,2\% em países de baixa renda. A incidência média de $1 \%$ normalmente é aceita no Brasil e em outros países da América Latina. ${ }^{2-4}$

O Brasil registra 2,8 milhões de nascidos vivo por ano, e estima-se que quase 29.000 novos casos de DCC ocorrem a cada ano. ${ }^{2}$ Aproximadamente 20 a 30\% dos recém-nascidos (RN) com doenças cardíacas morrem no período neonatal. DCCs são as malformações com o maior impacto na morbidade e mortalidade infantis, assim como nos custos com saúde pública. ${ }^{2,4,5}$

Múltiplos fatores ambientais e genéticos, como mutações, alterações cromossômicas e alterações genéticas, estão inter-relacionados e são a base das DCCs. Considerando a variedade de genes que coordenam o desenvolvimento do coração e os vários mecanismos que simultaneamente guiam o desenvolvimento geral do embrião, é razoável imaginar que as mudanças que interferem nesta organização complexa podem levar a várias malformações. Do ponto de vista genético, é importante avaliar se a DCC é uma ocorrência isolada ou se está associada a outras características, de forma a constituir uma síndrome. ${ }^{6}$ A SD22q11.2 é considerada como a segunda mudança cromossômica mais associada às DCCs, com incidência de 1:4000-5000 nascidos vivos, e aproximadamente $5 \%$ dos pacientes com doenças cardíacas apresentam esta deleção..$^{7-9}$ Anomalias conotruncais, principalmente a tetralogia de Fallot, atresia pulmonar com defeito do septo ventricular, transposição das grandes artérias, truncus arteriosus tipo 1 e arco aórtico interrompido (IAA) tipo B são as DCCs mais associadas à SD22q11.2..$^{8-10}$

É importante notar que outras deleções e/ou duplicações que podem ser encontradas em pacientes cardíacos e sua detecção permitem o acompanhamento multidisciplinar adequado, a avaliação do prognóstico e o aconselhamento genético.

\section{Pacientes e métodos}

\section{Pacientes}

Investigamos 118 recém-nascidos com DCC (69 meninos; idade média de 31,7 dias) hospitalizados para correção cirúrgica de maio de 2012 a junho de 2014. Foram incluídos consecutivamente da Unidade de Cardiologia Pediátrica do Instituto do Coração, no Hospital das Clínicas, Faculdade de
Medicina da Universidade de São Paulo (InCor - HCFMUSP), do Centro Neonatal do Instituto da Criança, Hospital das Clínicas, Faculdade de Medicina da Universidade de São Paulo (ICr - HCFMUSP), do Centro de Tratamento Intensivo Neonatal (CTIN2) do ICr-HCFMUSP.

O Comitê de Ética do HCFMUSP aprovou o estudo e todas as famílias dos pacientes assinaram um termo de consentimento.

\section{Extração de DNA}

O DNA genômico foi isolado de $3 \mathrm{~mL}$ de sangue periférico, utilizando um kit de isolamento de DNA disponível comercialmente (QIAamp DNA Blood Mini Kit ${ }^{\circledR}$, Qiagen, Hilden, Alemanha), de acordo com as instruções do fabricante. A qualidade e a quantidade das amostras de DNA foram determinadas utilizando um fluorímetro Qubit ${ }^{\circledR}$ 2.0 (Invitrogen, Carlsbad, Califórnia, EUA), e sua integridade foi verificada por meio da eletroforese em gel de agarose.

\section{Estudos da MLPA}

A técnica da amplificação multiplex de sondas dependente de ligação (MLPA) foi realizada com cinco kits diferentes: SALSA P064 para as síndromes de microdeleção e microduplicação mais comuns; P036 e P070 para desequilíbrio subtelomérico; e P356 e P250, específicos para o cromossomo 22q e DiGeorge e Velocardiofacial (MRC-Holland, Amsterdã, Holanda). As reações da MLPA foram realizadas de acordo com o protocolo do fabricante. Analisamos os resultados com o software GeneMarker ${ }^{\circledR}$ (Softgenetics, LLC, State College, Pa., EUA). Os resultados foram considerados anormais quando foram observados picos relativos, com tamanho menor que 0,75 (deleção) ou maior que 1,25 (amostras duplicadas em relação ao normal).

\section{Resultados}

A doença cardíaca congênita mais frequente foi a transposição das grandes artérias, observada em 11,9\% dos 118 recém-nascidos, seguida da coarctação da aorta, em 10,2\%; tetralogia de Fallot e comunicação intraventricular, em 9,3\%; e estenose pulmonar, em 7,6\%. Todos os defeitos cardíacos congênitos estão descritos na Tabela 1. Além da DCC, três desses pacientes tinham hipocalcemia, infecções recorrentes e dismorfismo facial.

Uma análise molecular pela MLPA detectou deleções típicas na 22q11.2 em 10 dos 118 recém-nascidos (8,5\%), sendo sete meninos e três meninas com idade de um a 330 dias. Todos os pacientes apresentaram manifestações típicas da doença. A Tabela 2 mostra os dados clínicos e os kits de MLPA que detectaram a 22q11.2.

Ao pesquisar a deleção 22q11.2 em pacientes com doenças cardíacas, a técnica da MLPA detectou outras microdeleções ou microduplicações em seis outros pacientes. Todos eram do sexo masculino, com idades variando de um a 44 dias. Os dados relacionados a esses pacientes com outras microduplicações ou microdeleções e os kits MLPA utilizados para o diagnóstico estão demonstrados na Tabela 3. 


\section{Artigo Original}

Tabela 1 - Defeitos cardíacos congênitos presentes nos 118 recém-nascidos incluídos

\begin{tabular}{|c|c|c|}
\hline Defeitos cardíacos congênitos & N & $\%$ \\
\hline $\begin{array}{l}\text { Átrio e grandes veias } \\
\text { - Drenagem venosa pulmonar anômala parcial } \\
\text { - Drenagem venosa pulmonar anômala total } \\
\text { - Isomerismo atrial direito } \\
\text { - Defeito do septo atrial }\end{array}$ & $\begin{array}{l}3 \\
3 \\
3 \\
3\end{array}$ & $\begin{array}{l}2,6 \\
2,6 \\
2,6 \\
2,6\end{array}$ \\
\hline $\begin{array}{l}\text { Conexão atrioventricular } \\
\text { - Atresia mitral } \\
\text { - Atresia tricúspide }\end{array}$ & $\begin{array}{l}2 \\
2\end{array}$ & $\begin{array}{l}1,7 \\
1,7\end{array}$ \\
\hline $\begin{array}{l}\text { Conexão ventriculoarterial } \\
\text { - Transposição das grandes artérias } \\
\text { - Dupla via de saída do ventrí́culo direito } \\
\text { - Dupla via de saída do ventrículo esquerdo } \\
\text { - Truncus arteriosus } \\
\text { - Dupla via de entrada do ventrículo esquerdo }\end{array}$ & $\begin{array}{c}14 \\
8 \\
4 \\
4 \\
1\end{array}$ & $\begin{array}{l}11,9 \\
6,8 \\
3,4 \\
3,4 \\
0,8\end{array}$ \\
\hline $\begin{array}{l}\text { Ventrículo } \\
\text { • Coração esquerdo hipoplásico }\end{array}$ & 5 & 4,2 \\
\hline $\begin{array}{l}\text { Septo ventricular } \\
\text { - Comunicação intraventricular }\end{array}$ & 11 & 9,3 \\
\hline $\begin{array}{l}\text { Fluxo no trato de saída do ventrículo direito } \\
\text { - Estenose pulmonar valvar } \\
\text { - Atresia pulmonar } \\
\text { - Estenose pulmonar }\end{array}$ & $\begin{array}{l}9 \\
6 \\
3\end{array}$ & $\begin{array}{l}7,6 \\
5,2 \\
2,6\end{array}$ \\
\hline $\begin{array}{l}\text { Tetralogia de Fallot e variantes } \\
\text { - Tetralogy de Fallot } \\
\text { - Atresia pulmonar com comunicação interventricular }\end{array}$ & $\begin{array}{c}11 \\
4\end{array}$ & $\begin{array}{l}9,3 \\
3,4\end{array}$ \\
\hline $\begin{array}{l}\text { Fluxo no trato de saída do ventrículo esquerdo } \\
\text { - Estenose aórtica valvar }\end{array}$ & 1 & 0,8 \\
\hline $\begin{array}{l}\text { Artéria coronária } \\
\text { - Origem anômala da artéria coronária esquerda }\end{array}$ & 1 & 0,8 \\
\hline $\begin{array}{l}\text { Conexões ventriculoarteriais } \\
\text { Coarctação da aorta } \\
\text { Arco aórtico interrompido tipo B } \\
\text { Estenose pulmonar }\end{array}$ & $\begin{array}{c}12 \\
4 \\
1\end{array}$ & $\begin{array}{l}10,2 \\
3,4 \\
0,8\end{array}$ \\
\hline
\end{tabular}

Tabela 2 - Dados clínicos e análise molecular de 10 pacientes com deleção 22q11.2 típica

\begin{tabular}{lccc}
\hline Paciente N. & Gênero & Idade no diagnóstico (meses) & Kits MLPA \\
\hline 11 & $\mathrm{M}$ & 6,7 & $\mathrm{P} 064, \mathrm{P} 250, \mathrm{P} 356$ \\
\hline 47 & $\mathrm{M}$ & 11 & $\mathrm{P} 064, \mathrm{P} 250, \mathrm{P} 356$ \\
\hline 53 & $\mathrm{M}$ & 0,1 & $\mathrm{P} 064, \mathrm{P} 250$ \\
\hline 67 & $\mathrm{M}$ & 0,5 & $\mathrm{P} 064, \mathrm{P} 250, \mathrm{P} 036$ \\
\hline 78 & $\mathrm{~F}$ & 4 & $\mathrm{P} 064, \mathrm{P} 250, \mathrm{P} 356$ \\
\hline 85 & $\mathrm{M}$ & 1,1 & $\mathrm{P} 064, \mathrm{P} 250$ \\
\hline 103 & $\mathrm{M}$ & 0,4 & $\mathrm{P} 064$ \\
\hline 106 & $\mathrm{M}$ & 1 & $\mathrm{P} 250, \mathrm{P} 356$ \\
\hline 115 & $\mathrm{M}$ & 9 & $\mathrm{P} 064, \mathrm{P} 250, \mathrm{P} 356$ \\
\hline 122 & $\mathrm{~F}$ & 2,5 & $\mathrm{P} 356$ \\
\hline
\end{tabular}

F: feminino; M: masculino. 
Tabela 3 - Dados clínicos e análise molecular de seis pacientes com outras alterações genômicas

\begin{tabular}{|c|c|c|c|c|c|}
\hline Paciente $\mathbf{N}$. & Gênero & Idade (dias) & Alteração genômica & Região cromossômica & Kits MLPA \\
\hline 1 & $\mathrm{M}$ & 4 & Deleção & $8 p 23$ & P250 \\
\hline 8 & $\mathrm{M}$ & 7 & Duplicação & $7 q 11.2$ & P036, P070 \\
\hline 15 & $\mathrm{M}$ & 13 & Deleção & $1 p 36$ & P064, P070 \\
\hline 34 & $\mathrm{M}$ & 44 & Duplicação & $12 p$ & P036 \\
\hline 51 & $\mathrm{M}$ & 1 & Duplicação & $8 q 24$ & P070 \\
\hline 61 & $M$ & 1 & Deleção & $8 p 23$ & P250 \\
\hline
\end{tabular}

A deleção 8p23 foi confirmada pelo kit 250, que é específico para a região crítica da síndrome de deleção 22q11.2, mas também permite o diagnóstico molecular em outros locais genômicos, como 4q35.2, 8p23.1, 9q34. 3, 10p12.31, 10p14, 10p15.1 e 17p13.3.

Detectamos alterações subteloméricas utilizando os kits P036 e P070 em um caso, e o paciente apresentou a duplicação $7 q$. Houve mudanças em somente um dos kits subteloméricos. Uma análise molecular mostrou que, em um deles, o kit P036 encontrou a duplicação de 12p, e no outro, o kit P070 encontrou a duplicação de 8q24.

Entre os casos deste estudo, o kit P064 permitiu a identificação de mudanças em outras regiões suscetíveis a rearranjos, como a microdeleção do 1 p36.

A triagem MLPA em crianças com DCC levou à detecção precoce do defeito genético em 16 recém-nascidos; dez com deleção 22q11.2 e seis outros (4,2\%), conforme segue: deleção 8p23 em dois recém-nascidos; duplicação 8q24 em um recém-nascido; duplicação 7q, duplicação 12p e deleção 1 p36.

\section{Discussão}

É importante enfatizar que o diagnóstico precoce da DCC tem crescido devido ao aprimoramento do diagnóstico pré-natal. A literatura internacional mostra que 43,6\% dos pacientes cardíacos são diagnosticados na primeira semana de vida; $70 \%$, até seis meses de idade; e até $86 \%$ em no máximo dois anos. ${ }^{11-13}$

Como as doenças cromossômicas são mais frequentes em pacientes com malformações cardíacas do que na população em geral, é essencial enfatizar a importância da investigação genética no diagnóstico da DCC. O diagnóstico molecular específico de uma síndrome genética vai permitir um acompanhamento multidisciplinar adequado, uma avaliação prognóstica e a conscientização da família sobre os riscos da recorrência. ${ }^{14-16}$

As síndromes da microdeleção e/ou da microduplicação associadas à doença cardíaca congênita não são detectadas no exame clássico do cariótipo; elas podem ser somente identificadas quando utilizamos outras técnicas moleculares, como hibridação in situ por fluorescência (FISH), MLPA ou, mais recentemente, análise cromossômica por microarray (CMA) ${ }^{11}$
Este estudo demonstrou a importância de se utilizar a técnica da MLPA para o diagnóstico precoce da SD22q11.2 em pacientes com DCCs no primeiro ano de vida. Observamos que esta deleção foi detectada em 8,5\% dos pacientes, o que excedeu as taxas descritas na literatura. Este achado pode estar relacionado às características dos pacientes incluídos no estudo, já que a maioria recebia tratamento em um hospital terciário nacional de referência. ${ }^{9,10,16-23}$

Apesar dos avanços recentes e do fato de a SD22q11.2 ser considerada como a microdeleção cromossômica mais comum em humanos, de acordo com estudos realizados na Europa e nos Estados Unidos, ${ }^{8}$ esta síndrome permanece subdiagnosticada no Brasil devido à falta de informação entre médicos e à dificuldade de se obter uma triagem específica no Sistema Único de Saúde (SUS). No Brasil, não há estudos sobre a triagem da deleção 22q11.2 utilizando a técnica da MLPA exclusivamente na faixa etária que nós analisamos. Neste período da vida, o fenótipo nem sempre é claro, o que dificulta o diagnóstico precoce nesses pacientes. . $^{8,9,12}$

Nosso objetivo é enfatizar que as doenças cardíacas congênitas devem ser um sinal de alerta para a SD22q11.2, especialmente em pacientes com malformações conotruncais, hipocalcemia e dismorfismo facial. Porém, ainda é um grande desafio devido aos seus vários aspectos clínicos e comportamentais, que podem atrasar o diagnóstico e o tratamento adequado. Além disso, a importância de se detectar outras deleções e/ou duplicações relacionadas à DCC é clara, já que isso levaria ao aconselhamento genético, ao acompanhamento multidisciplinar e à identificação de outras malformações associadas. ${ }^{10,12,16,22-27}$

Os resultados descritos aqui estão alinhados com a literatura, que confirma a presença das malformações conotruncais na maioria dos pacientes com deleção 22q11.2. ${ }^{9,16-18,23,28-30}$

Pacientes com SD 22q11.2 e DCC severa provavelmente morrem antes de ter acesso ao hospital terciário de referência, levando à subnotificação da sua taxa de ocorrência. É importante notar que, no Brasil, diferentemente de países desenvolvidos, o aborto em casos de malformação não é permitido por lei. Isso também justificaria a diferença quando comparamos nossa série de casos com as taxas de detecção de deleção 22q11.2 descritas na literatura. 9,10,17,19,20 


\section{Artigo Original}

Neonatologistas, cardiologistas pediátricos e cirurgiões cardíacos devem estar cientes das especificidades e do cuidado associados à SD22q11.2. Como se pode observar, pacientes afetados pela condição normalmente apresentam mudanças em vários sistemas do corpo e requerem muitas intervenções clínicas, envolvendo hospitalização ao longo da vida. Deve-se notar que pacientes com SD22q11.2 têm taxas de mortalidade mais altas em comparação a outras síndromes genéticas (como a Síndrome de Down) e casos não-sindrômicos de DCC. ${ }^{16,23,31-33}$

Dada a importância desta síndrome altamente complexa, é essencial fazer uma triagem e detectá-la cedo no primeiro ano de vida, com base em um conjunto de características clínicas e laboratoriais. ${ }^{12}$ Vale notar que, neste grupo etário, as taxas de morbidade e mortalidade são altas, principalmente devido a infecções recorrentes resultando de aplasia ou hipoplasia tímica em DCC. ${ }^{12,34,35}$

Dentre os pacientes avaliados, a deleção 8p23 foi observada em dois deles. As doenças complexas foram dupla via de saída do ventrículo direito e hipoplasia tricúspide no ventrículo direito, ambas diagnosticadas na primeira semana de vida. É importante salientar que esses pacientes não apresentavam nenhuma anomalia clínica típica.

Vários autores sugeriram que a deleção $8 p$ pode ser mais frequente do que o número relativamente baixo de casos descritos na literatura. Os resultados dos estudos da correlação genético-fenótipo de indivíduos com DCC enfatizam a região cromossômica 8p23.1 como a região crítica para a morfogênese cardíaca. A porção proximal da região p23.1 contém o gene da proteína de ligação 4 (GATA4), que codifica um fator de transcrição que tem papel essencial no desenvolvimento dos corações humanos. CCs neste tipo de deleção podem ser explicadas pela haploinsuficiência do GATA4..$^{36-38}$

A deleção 8p23.1 pode estar associada a uma ampla gama de anomalias clínicas, incluindo dismorfismo facial, microcefalia, restrição de crescimento intrauterino, doenças psiquiátricas, déficit intelectual e doenças cardíacas, sendo que as mais frequentes são defeitos do septo atrioventricular e do septo atrial. ${ }^{36,38,39}$ Há relatos de deleção intersticial e terminal no braço curto do cromossomo 8 (mais frequentemente com rupturas nas regiões 8 p21 a 8p23), assim como deleções combinadas com duplicações. A variável status clínico se deve à extensão da deleção ou à localização do ponto de ruptura. ${ }^{38}$

O déficit intelectual é o achado mais frequente para este tipo de deleção. Aparentemente há uma relação entre o tamanho da região da deleção no cromossomo 8 e o nível de deficiência intelectual, segundo a qual as deleções terminais mais distais à região 23.1 estão associadas ao dano intelectual menor. ${ }^{36,40}$

Também foi possível observar déficit motor, atraso linguístico e mudanças comportamentais (hiperatividade, déficit de atenção e agressividade). As deleções SOX7 também têm um papel no atraso do desenvolvimento e, possivelmente, nas características dismórficas de indivíduos com deleção 8p23. 38,41,42

Este estudo detectou a duplicação $7 q 11.2$ em um paciente. Diferentemente da deleção de 1p36 e 8p23, este tipo de alteração genômica não tem correlação com doenças cardíacas. O paciente incluído no estudo tinha coarctação da aorta, e o diagnóstico foi feito aos sete dias de vida; não havia nenhuma malformação aparente.

É importante notar que esta é uma patologia rara, já que há aproximadamente 54 casos descritos na literatura. Duplicações parciais $7 q$ foram classificadas em quatro grupos de acordo com a região cromossômica afetada. ${ }^{43}$

Para o paciente detectado com a duplicação 8p24, haverá um acompanhamento neurológico para monitorar o desenvolvimento clínico, já que os genes associados à epilepsia estão localizados na região 8p24, e alterações nesta região podem ser responsáveis por convulsões tônicas, às vezes seguidas de convulsões clônicas generalizadas. Um dos genes envolvidos é o KCNQ3 (canais de potássio dependentes de voltagem, subfamília $\mathrm{Q}$, membro 3). O desenvolvimento costuma ser benigno, com remissão no primeiro ano de vida. É importante observar que neste tipo de alteração genômica não há maior correlação com alguns tipos específicos de doenças cardíacas. O paciente da nossa série tinha atresia pulmonar e nenhum outro achado clínico. ${ }^{44}$

A outra alteração genômica detectada em um paciente com IAA foi a duplicação 12p. A literatura demonstra que esta alteração genômica está relacionada à síndrome de Pallister-Killian (SPK), pela qual pacientes apresentam alterações craniofaciais típicas, defeitos cardíacos congênitos, atraso de desenvolvimento variável, dano intelectual, convulsões, hipotonia, surdez, lóbulos da orelha grandes, pescoço curto, mãos grandes com dedos pequenos, encurtamento desproporcional de braços e pernas, deficiências de pigmentação da pele, hérnia diafragmática congênita e outras anomalias sistêmicas. A SPK está relacionada à trissomia ou à tetrassomia de genes específicos localizados na região duplicada. O fenótipo SPK normalmente é observado em indivíduos com duplicações completas ou parciais de $12 p$ (trissomia 12p, em vez de tetrassomia 12p) como resultado da duplicação intersticial ou translocação desequilibrada. ${ }^{45}$

Nesses casos, a correlação fenótipo-genótipo é muito importante para o diagnóstico clínico e as abordagens terapêuticas. Porém, alguns fenótipos parecem estar associados a duplicações de regiões cromossômicas específicas.

Avanços médicos permitiram mais precisão na correlação entre a estrutura do DNA e o fenótipo clínico. Como demonstrado neste estudo, as técnicas citogenéticas permitem o diagnóstico precoce de diferentes síndromes de deleção e duplicação em pacientes cardíacos. Deve-se notar que um conhecimento aprofundado de testes citogenéticos e sua aplicação em diferentes campos da medicina levarão à escolha adequada para a triagem: MLPA, array, painel ou sequenciamento de exoma.

Estudos recomendam que todos os recém-nascidos ou crianças com doenças cardíacas e dismorfismo, ou outras anomalias congênitas, sejam testadas para deleção 22q11.2. Além disso, os desequilíbrios genotípicos de outras regiões cromossômicas, incluindo 10p12-p15, 4q21-q35, 8p21-p23, 17 p13 e 18q21, podem ser encontrados em pacientes com suspeita clínica de SD22q11.2 e sem a deleção 22q11.2. ${ }^{46,47}$ 
A investigação genômica é crucial para realizar um perfil correto e precoce da etiologia de pacientes cardíacos, além de permitir o melhor entendimento da alta variabilidade fenotípica nesses pacientes.

Por fim, acreditamos que este estudo vai ajudar a demonstrar a importância da investigação citogenética precoce em pacientes cardíacos para avaliar o prognóstico e o risco de recorrência, ao mesmo tempo em que leva ao tratamento correto, acompanhamento e aconselhamento familiar.

\section{Conclusões}

Os autores demonstraram a importância de investigar e detectar alterações genômicas utilizando a técnica da MLPA em recém-nascidos e crianças com doenças cardíacas. O rápido progresso do uso de testes citogenéticos no ambiente clínico leva os médicos a gerenciar as DDCs e considerar que muitos pacientes podem sofrer de déficits cromossômicos ou até genéticos.

A detecção de alterações citogenômicas permitirá que médicos avaliem o prognóstico e o risco de recorrência, além de permitir o devido acompanhamento e tratamento, com o objetivo de melhorar a qualidade de vida das crianças.

\section{Referências}

1. Lima AF, Santos KS, Oliveira SG, Rocha TACS, Fermoseli AFO Incidência de malformações congênitas em recém-nascidos em maternidade pública da capital de Alagoas, BR. Interfaces Científicas - Saúde e Ambiente.2018;6(3):103-14.

2. Brasil. Ministério da Saúde (2017) Síntese de evidências para políticas de saúde: Diagnóstico precoce de cardiopatas congênitas. Ministério da Saúde, Secretaria de Ciência, Tecnologia e Insumos Estratégicos, Departamento de Ciência e Tecnologia. Brasília`;2017.

3. Mitchell SC, Korones SB, Berendes HW (1971) Congenital heart disease in 56,109 births. Incidence and natural history. Circulation.1971;43(3):323-32.

4. Rosa RCM, Rosa RFM, Zen PRG, Paskulin GA. Cardiopatias congênitas e malformações extracardíacas. Rev Paul Pediatr.2013; 31(2): 243-51

5. Caneo If, Jatene MB, Yatsuda N, Gomes WJ. Uma reflexão sobre o desempenho da cirurgia cardíaca pediátrica no Estado de São Paulo. Rev Bras Cir Cardiovasc. 2012;27(3):457-62

6. Rimoin, David L Emery and Rimoin's principles and practice of medical genetics, 6th ed. Philadelphia: Elsevier/Churchill Livingstone Elsevier, 2013.

7. Ikari NM, Azeka E, Aiello VD, Atik E, Barbero-Marcial M, Ebaid M. Uhl's anomaly. Differential diagnosis and indication for cardiac transplantation in an infant. Arq Bras Cardiol.2001;77(1):73-6

8. Kobrynski L, Sullivan K. Velocardiofacial syndrome, DiGeorge syndrome: the chromosome 22q11.2 deletion syndromes. Lancet. 370(9596):1443-52.

9. Gennery AR. Immunological aspects of 22q 11.2 deletion syndrome. Cell Mol Life Sci.2012; 69:17-27.

10. Goldmuntz E. DiGeorge Syndrome: New Insights. Clin Perinatol. 2005;32(10):963-3.

11. Miller DT, Adam MP, Aradhya S, Biesecker LG, Brothman AR, Carter NP, et al. Consensus Statement: Chromosomal Microarray Is a First-Tier Clinical Diagnostic Test for Individuals with Developmental Disabilities or Congenital Anomalies. Am J Hum Genet. 86(5):749-64

\section{Contribuição dos autores}

Concepção e desenho da pesquisa: Grassi MS, Pastorino AC, Kim C, Kulikowski L, Carneiro-Sampaio M; Obtenção de dados: Grassi MS, Pastorino AC, Dorna MB, Jatene M, Miura N, Kulikowski L, Carneiro-Sampaio M; Análise e interpretação dos dados: Grassi MS, Montenegro M, Zanardo EA, Pastorino AC, Dorna MB, Kulikowski L, Carneiro-Sampaio M; Análise estatística e Redação do manuscrito: Grassi MS, Pastorino AC, Kulikowski L, Carneiro-Sampaio M; Obtenção de financiamento: Pastorino AC, Kim C, Kulikowski L, Carneiro-Sampaio M; Revisão crítica do manuscrito quanto ao conteúdo intelectual importante: Pastorino AC, Kim C, Kulikowski L, Carneiro-Sampaio M.

\section{Potencial conflito de interesse}

Não há conflito com o presente artigo.

\section{Fontes de financiamento}

O presente estudo foi financiado pela FAPESP (grant 2014/50489-9).

\section{Vinculação acadêmica}

Este artigo é parte de tese de doutorado de Marília Sierro Grassi pela Universidade de São Paulo.

12. Grassi MS, Jacob CMA, Kulikowski LD, Pastorino AC, Dutra RL, Miura N et al. Congenital Heart Disease as a Warning Sign for the Diagnosis of the 22q11.2 Deletion. Arq Bras Cardiol.2014;103(5):382-90.

13. Stephensen SS, Sigfusson G, Eiriksson H, Sverrisson JT, Torfason B, Haraldsson A, et al. Congenital cardiac malformations in Iceland from 1990 through 1999. Cardiol Young .2004;14(4):396-401.

14. Ionita-Laza I, Rogers AJ, Lange C, Raby BA, Lee C. Genetic association analysis of copy-number variation (CNV) in human disease pathogenesis. Genomics.2009;93(1):22-6.

15. Molck MC, Simioni M, Paiva Vieira T, Sgardioli IC, Paoli Monteiro F, Souza J, et al. Genomic imbalances in syndromic congenital heart disease. J Pediatr. (Rio J) 93(5):497-507.

16. McDonald-McGinn DM. 22q11.2 deletion syndrome: a tiny piece leading to a big picture. Am J Med Genet. 2018;176(10):2055-7.

17. Fomin AFB, Pastorino AC, Kim CA, Pereira CA, Carneiro-Sampaio M Abe-Jacob CM DiGeorge Syndrome: a not so rare disease. Clinics. (São Paulo).2010; 65:865-9.

18. Zhang X, Xu Y, Liu D, Geng J, Chen S, Jiang Z, Fu Q, Sun K. A modified multiplex ligation-dependent probe amplification method for the detection of $22 \mathrm{q} 11.2$ copy number variations in patients with congenital heart disease. BMC Genomics. 2015:16:364.

19. Mlynarski EE, Xie M, Taylor D, Sheridan MB, Guo T, Racedo SE, et al International Chromosome 22q11.2 Consortium (2016) Rare copy number variants and congenital heart defects in the $22 \mathrm{q} 11.2$ deletion syndrome. Hum Genet.2016;135:273-85.

20. Guo T, Repetto GM, McDonald McGinn DM, Chung JH, Nomaru H Campbell CL, et al. International 22q11.2 Consortium/Brain and Behavior Consortium. Genome-Wide Association Study to Find Modifiers for Tetralogy of Fallot in the 22q11.2 Deletion Syndrome Identifies Variants in the GPR98 Locus on 5q14.3. Circ Cardiovasc Genet.2017;10(5): https://doi: 10.1007/ s00439-015-1623-9 
21. Kruszka P, Addissie YA, McGinn DE, Porras AR, Biggs E, Share M, et al. 22q11.2 deletion syndrome in diverse populations. Am J Med Genet. A173(4):879-88.

22. Mutlu ET, Aykan HH, Karagoz T. Analysis of gene copy number variations in patients with congenital heart disease using multiplex ligation-dependent probe amplification. Anatol J Cardiol.2018;20(1):9-15.

23. Sullivan K. Chromosome 22q11.2 deletion syndrome and DiGeorge syndrome. Immunol Rev. 2019;287(1):186-201.

24. Matsuoka R, Kimura M, Scambler P, Morrow B, Imamura S, Minoshima S, et al. Molecular and clinical study of 183 patients with conotruncal anomaly face syndrome. Hum Genet .1998;103:7080.

25. McDonald-McGinn D, Kirschner R, Goldmuntz E, Sullivan K, Eicher P, Gerdes M,et al. The Philadelphia story: the 22q11.2 deletion: report on 250 patients. Genet Couns.1999;10(1):11-21.

26. Karayiorgou M, Simon TJ, Gogos JA. 22q11.2 microdeletions: linking DNA structural variation to brain dysfunction and schizophrenia. Nat Rev Neurosci.2010; 11(6):402-16.

27. Maggadottir SM, Sullivan KE. The diverse clinical features of chromosome 22q11.2 deletion syndrome (DiGeorge Syndrome). J Allergy Clin Immunol Pract.2013;10(1):10(1):589-94

28. Campbell IM, Sheppard S, Crowley TB, McGinn D, Bailey A, McGinn MJ, et al. What is new 22q? An update from the 22q and you Center at the Children`s Hospital of Philadelphia. Am J Med Genet A 2018;176:2058-69.

29. Cohen JL, Crowlwy TB, McGinn DE, McDougall C, Unolt M, Lambert MP, et al. 22q and two: 22q11.2 deletion syndrome and coexisting conditions. Am J Med Genet.2018; 176(10):2203-14.

30. Morrow BE, McDonald-McGinn D, Emanuel BS, Vermeesch JR, Scambler PJ. Molecular genetics of 22q11.2 deletion syndrome. Am J Med Genet A.2018;176(10):2070-81.

31. Carotti A, Digilio MC, Piacentini G, Saffirio C, Di Donato RM, Marino B. Cardiac defects and results of cardiac surgery in 22q11.2 deletion syndrome. Dev Disabil Res Rev.2018; 14(1):35-42.

32. Formigari R, Michielon G, Digilio MC, Piacentini G, Carotti A, Giardini A, et al. Genetic syndromes and congenital heart defects: how is surgical management affected? Eur J Cardiothorac Surg.2009;35(4):606-14.

33. Palmer LD, Butcher NJ, Boot E, Hodgkinson KA, Heung T, Chow EWC, et al. Elucidating the diagnostic odyssey of 22q11.2 deletion syndrome. Am J Med Genet A.2018;176(4):936-44.

34. Rosa RF, Pilla CB, Pereira VL, Flores JA, Golendziner E, Koshiyama DB, et al. 22q11.2 deletion syndrome in patients admitted to a cardiac pediatric intensive care unit in Brazil. Am J Med Genet A.2008;146A(13):1655-61.
35. Rosa RFM, Trevisan P, Koshiyama DB, Pillsa CB, Zens PRG, Varella-Garcia M, et Síndrome de deleção 22q11 e cardiopatias congênitas complexas. Rev Assoc Med Bras. 1992;57(1):62-5.

36. Hutchinson R, Wilson M, Voullaire L (1992) Distal 8p deletion (8p23.1---8pter): a common deletion? J Med Genet, 29(6):407-11.

37. Bhatia SN, Suri V, Bundy A, Krauss CM. Prenatal detection and mapping of a distal $8 p$ deletion associated with congenital heart disease. Prenat Diagn.1999;19(9):863-7.

38. Páez MT, Yamamoto T, Hayashi K, Yasuda T, Harada N, Matsumoto N, et al. Two patients with atypical interstitial deletions of 8p23.1: mapping of phenotypical traits. Am J Med Genet A. 2008; 146A(9):1158-65.

39. Wu BL, Schneider GH, Sabatino DE, Bozovic LZ, Cao B, Korf BR. Distal 8p deletion (8)(p23.1): an easily missed chromosomal abnormality that may be associated with congenital heart defect and mental retardation. Am J Med Genet.1996;62(1):77-83.

40. Gilmore L, Cuskelly M, Jobling A, Smith S. Deletion of 8p: a report of a child with normal intelligence. Dev Med Child Neurol.2001;43(12):843-6.

41. Digilio MC, Giannotti A, Marino B, Dallapiccola B (1993) Atrioventricular canal and 8p- syndrome. Am J Med Genet 47:437-438

42. Long F, Wang X, Fang S, Xu Y, Sun K, Chen S, Xu R(2013) A potential relationship among beta-defensins haplotype, $\mathrm{SOX} 7$ duplication and cardiac defects. PLoS One 8:e72515

43. Ruiz-Botero F, Pachajoa H (2016) Deletion 21q22.3 and duplication 7q35q36.3 in a Colombian girl: a case report. J Med Case Rep.1993;10:204.

44. Hu J, Sathanoori M, Kochmar S, Azage M, Mann S, Madan-Khetarpal S, et al. A novel maternally inherited $8 \mathrm{q} 24.3$ and a rare paternally inherited $14 \mathrm{q} 23.3$ CNVs in a family with neurodevelopmental disorders. Am J Med Genet A. 2015;167A(8):1921-6.

45. Izumi K, Conlin LK, Berrodin D, Fincher C, Wilkens A, Haldeman-Englert C, Saitta SC, Zackai EH, Spinner NB, Krantz ID (2012) Duplication 12p and Pallister-Killian syndrome: a case report and review of the literature toward defining a Pallister-Killian syndrome minimal critical region. Am J Med Genet A. 2012;158A(12):3033-45.

46. Ziolkowska L, Kawalec W, Turska-Kmiec A, Krajewska-Walasek M, Brzezinska-Rajszys G, Daszkowska J, et al. Chromosome 22q11.2 microdeletion in children with conotruncal heart defects: frequency, associated cardiovascular anomalies, and outcome following cardiac surgery. Eur J Pediatr.2008;167(10):1135-40.

47. MolckMC, Monteiro FP, Simioni M, Gil-da-Silva-Lopes VL. 8p23.1 Interstitial Deletion in a Patient with Congenital Cardiopathy, Neurobehavioral Disorders, and Minor Signs Suggesting 22q11.2 Deletion Syndrome. J Dev Behav Pediatr.2015;36(7):544-8. 